\section{In vivo photoacoustic imaging of osteosarcoma in a rat model}

\author{
Jun $\mathrm{Hu}^{\mathrm{a}, *}$ Menglei $\mathrm{Yu}^{\mathrm{a}, *}$ Fei Ye, ${ }^{\mathrm{b}}$ and Da Xing ${ }^{\mathrm{b}}$ \\ a Shantou University-Medical College, The Department of \\ Orthopaedic of The First Affiliated Hospital, Shantou 515041, China \\ ${ }^{\text {b}}$ South China Normal University, College of Biophotonics, MOE Key \\ Laboratory of Laser Life Science and Institute of Laser Life Science, \\ Guangzhou 510631, China
}

\begin{abstract}
Osteosarcoma is one of the most common primary malignant tumors of the bone and the second leading cause of cancer-related deaths in the pediatric age group. Confirmed diagnosis and prompt treatment of osteosarcoma are critical for effective prognosis. In this study, we investigate the application of photoacoustic imaging (PAI) for the detection of osteosarcoma in an animal model. Crosssection images of a normal rat leg and a tumorous rat leg were successfully reconstructed in vivo. Morphological changes and the development of the implanted osteosarcoma were accurately mapped with time-dependent photoacoustic images. Furthermore, we evaluate the use of gold nanorods as contrast agents for imaging osteosarcoma with PAI. This is the first study that uses PAI to detect osteosarcoma in vivo, and the results suggest that PAI has the potential clinical application for detecting osteosarcoma in the early stage. $\odot 2011$ Society of Photo-Optical Instrumentation Engineers (SPIE). [DOI: 10.1117/1.3544502]
\end{abstract}

Keywords: photoacoustics; osteosarcoma; optical inspection; gold nanorods.

Paper 10525LR received Oct. 2, 2010; revised manuscript received Nov. 3, 2010; accepted for publication Dec. 30, 2010; published online Feb. 17, 2011.

\section{Introduction}

Osteosarcoma is one of the most common primary malignant tumors of the bone and the second leading cause of cancerrelated deaths in the pediatric age group. More than $80 \%$ of these tumors tend to occur in the long bones of the appendicular skeleton, which are undergoing rapid growth. ${ }^{1}$ Amputation has been the conventional treatment for all patients with osteosarcoma. However, the cure rate is less than $10 \%$ and almost all patients died within a year of diagnosis. Although this type of malignant tumor is one of the leading causes of death and morbidity, the therapeutic effects of osteosarcoma treatment is still faced with limits. There are several methods for detecting osteosarcoma in clinics (e.g., plain radiography, computed tomography, magnetic resonance imaging, angiography, dynamic bone scintigraphy, and if necessary, the type of reconstruction), but no method has been able to satisfactorily detect osteosarcoma in the early stage. ${ }^{2-5}$ Most patients died within 1 to 2 years after disease diagnosis. ${ }^{2}$ Obviously, the progress of

\footnotetext{
*Equally contributed to this work.
}

Address all correspondence to: Da Xing, Ph.D, Professor, South China Normal University, College of Biophotonics, MOE Key Laboratory of Laser Life Science \& Institute of Laser Life Science, Guangzhou 510631, China. Tel: + 86-20-85210089; Fax: + 86-20-8521-6052; E-mail: xingda@scnu.edu.cn therapeutic effects is largely based on a confirmed diagnosis in the early stage and whether the tumor is localized. New insights and novel imaging modalities are urgently needed for osteosarcoma detection.

Photoacoustic imaging (PAI) is a new imaging technology and a potential ideal candidate for clinical biomedical imaging, which is a hybrid technique that combines the high intrinsic contrast of optical imaging with the high spatial resolution of pure ultrasound imaging. ${ }^{6,7}$ PAI has been applied to in vivo imaging of vasculature in small animals and humans, ${ }^{8-11}$ and for detecting early-stage breast tumors or cancer. ${ }^{12,13}$ In this study, we established osteosarcoma models in rats and investigated the feasibility of detecting osteosarcoma using PAI in vivo. The different stages of osteosarcoma were visualized with photoacoustic (PA) images. Gold nanorods also were evaluated as a contrast agent for in vivo osteosarcoma imaging with PAI. As an essential step toward human body diagnosis, the feasibility of PAI for imaging osteosarcoma in vivo was verified through the study of the rat osteosarcoma model.

\section{Material and Methods}

\subsection{PA Experimental Setup}

The PA experimental setup of the in vivo imaging of rat osteosarcoma models is shown in Fig. 1. A tunable pulsed laser source (Vibrant B 532I, Opotek, California), with an operating wavelength of 532 and 690 to $960 \mathrm{~nm}$, a pulse width of $10 \mathrm{~ns}$, and a pulse repetition rate of $10 \mathrm{~Hz}$, was used to provide optical illumination for PAI. The laser beam was filtered by a variable slit to obtain a rectangular cross-homogeneous light and then focused into a line to illuminate into the sample with an optical cylindrical lens (focal length: $100 \mathrm{~mm}$ in the vacuum). The incident energy density on the leg surface was set at $8 \mathrm{~mJ} / \mathrm{cm}^{2}$. The rat body was fixed in a special box, and the toes of the rat were also clamped at the bottom of the water tank, which left the rat leg vertically exposed for pulse radiation. The body and leg can be scanned circularly around their axis, and controlled by a precise stepper motor in the horizontal $X-Y$ plane. To cover a 360 deg receiving angle for imaging the rat leg, a total of 50 steps with a constant 7.2 deg interval were taken. The transducer array with 64 elements was used to collect PA signals. The central frequency of the linear multielement transducer is $7.5 \mathrm{MHz}$ with a bandwidth of $70 \%$. Cross-section images of a normal and a tumorous rat leg were acquired at wavelength of $1064 \mathrm{~nm}$, and the images with injected gold nanorods were acquired at wavelength of $800 \mathrm{~nm}$.

\subsection{Osteosarcoma Rat Model and Experiment Operation}

Four-week-old male Sprague-Dawley (SD) rats were purchased from the laboratory animal center of Sun Yat-sen University. The UMR 106 osteosarcoma cell line syngeneic to SD rats was purchased from the American Type Culture Collection (ATCC; Manassas, Virgina) and cultured in RPMI-10\% FBS (Sigma) supplemented with L-glutamine $(2 \mathrm{mM})$, penicillin (100 U/ml), and streptomycin $(100 \mu \mathrm{g} / \mathrm{ml})$, and confirmed to be mycoplasma-free by routine testing. The number of cells was

1083-3668/2011/16(2)/020503/3/\$25.00 @ 2011 SPIE 


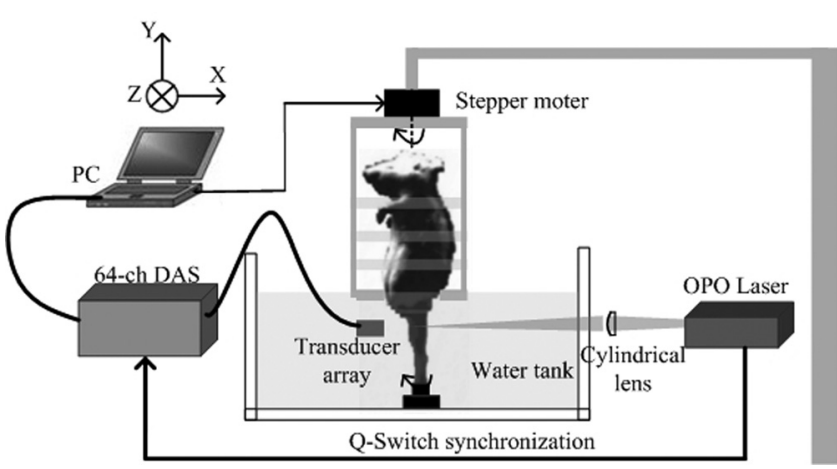

Fig. 1 Schematic representation of the PAI system for in vivo imaging of osteosarcoma on an animal model.

adjusted to about $2 \times 10^{7} / \mathrm{ml}$, and inoculated directly into the proximal part of the tibial shaft surface of the anesthetized SD rats with a syringe and 25-gauge needle. After implantation, the rats were treated with cyclosporin A $(10 \mathrm{mg} / \mathrm{kg})$ every day until 1 week after implantation. ${ }^{14}$ Before imaging, the fur over the leg of the rat was shaved and chemically depilated. General anesthesia (sodium pentobarbital, $40 \mathrm{mg} / \mathrm{kg}$, i.p.) was administered to keep the rat motionless throughout the experiment.

To further explore the PAI detection of osteosarcoma, gold nanorods were used as a contrast agent to enhance the imaging of the tumor area after the tumor was inoculated 10 days in the rat leg. Gold nanorods with a mean aspect ratio of 3.9, which were purchased from Strem Chem., Inc., exhibited peak optical absorption at $808 \mathrm{~nm}$. During PAI experiments, injections were administered subcutaneously through a needle via four opposite directions. For every injection, $10 \mu \mathrm{l}$ agent with a gold nanorod concentration of $10^{9}$ nanorods $\mathrm{ml}^{-1}$ was introduced. PAI experiments before and after injections were performed as described above.

\section{Results}

A PA-reconstructed cross-section image of a normal rat leg was successfully obtained with the skin intact, as shown in Fig. 2(a). The reconstructed PA image corresponds to the transverse section of the rat leg, where the tissue configuration is evidently visualized. The tissue structure of the reconstructed PAI corresponds with the anatomical photograph of the leg shown in Fig. 2(b). By comparison with the anatomical photo, the bone, blood, tissue, and tissue fat are marked with the arrowheads, respectively. The experiment showed that PAI can be used for the noninvasive imaging of sections of a rat leg.

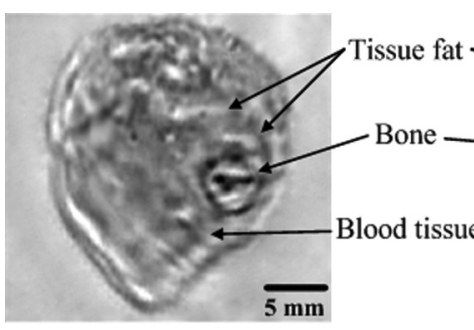

(a)

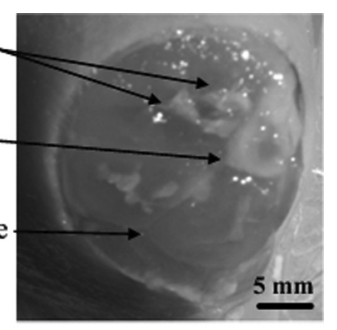

(b)
Fig. 2 Noninvasive in vivo imaging of a cross-section of a normal rat leg. (a) PA image of a rat leg acquired with the skin intact. (b) Anatomical photograph of a cross- section of a normal rat leg.

PA images of the tumorous rat leg at 5, 10, and 15 days after the inoculation of UMR-106 osteosarcoma cells are shown in Figs. 3(a), 3(b) and 3(c), respectively. Compared with the reconstructed PA images, the morphology of the rat leg changes gradually after the tumor has been implanted. The area marked with the arrowhead shows evident protrusion 15 days after implantation, and is considered a highly suspicious tumor region. After the experiment, the rat was subjected to necropsy. A histopathological test with hematoxylin-eosin staining demonstrated an implanted osteosarcoma. The implanted tumor was characterized by fusiform or triangle cells [inset of Fig. 3(d)] along nutrient vessels with nuclear pleomorphism, mitoses, bone invasion, and necrosis. The tumor was unequivocally detected with PAI and matched well with the histological photograph both in position and shape. The images suggest that PAI can provide information on tumor growth changes and osteosarcoma tissues in the leg.

Gold nanorods as a contrast agent were tested to enhance PAI detection of osteosarcoma in the rat model. The image in Fig. 4(a) was taken before the administration of gold nanorods, whereas the images in Figs. 4(b) and 4(c) were taken $30 \mathrm{~min}$ and $6 \mathrm{~h}$ after the administration of the contrast agent, respectively. The tumor cells were designedly inoculated in the upside of the rat leg as marked by the arrow in Fig. 4(a), but there is no obvious morphological change from the figure. Injections of gold nanorods were administered subcutaneously through a needle via four directions, as indicated by white arrows in Fig. 4(b). Thus, the image taken $30 \mathrm{~min}$ after the administration of gold nanorods shows an enhanced signal volume in the injection sites. The higher signal distribution represents higher concentrations of gold nanorods. The PA image taken $6 \mathrm{~h}$ after the administration of gold nanorods shows a local enhancement, suggesting the tumor-implanted region. The photoacoustic enhancement on the

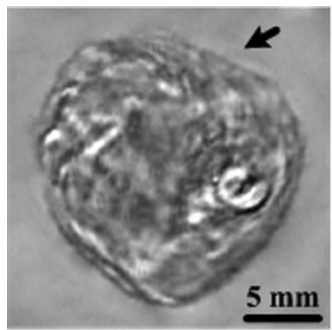

(a)

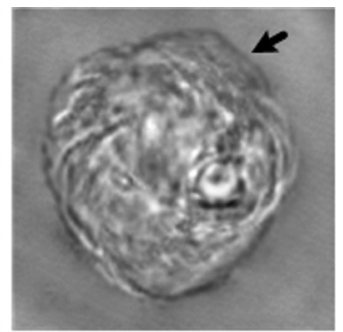

(b)

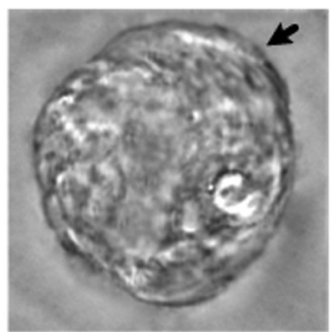

(c)

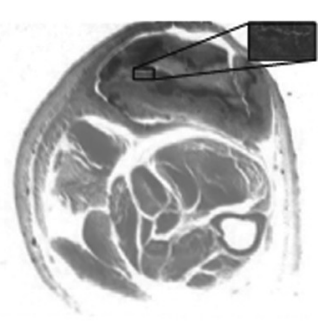

(d)

Fig. 3 Noninvasive in vivo photoacoustic imaging of osteosarcoma in a rat leg (a) 5 days, (b) 10 days, and (c) 15 days after tumor inoculation. (d) Histological image of a cross-section of the rat leg 15 days after PAI experiments. Tumor suspicious area is indicated by an arrow. 


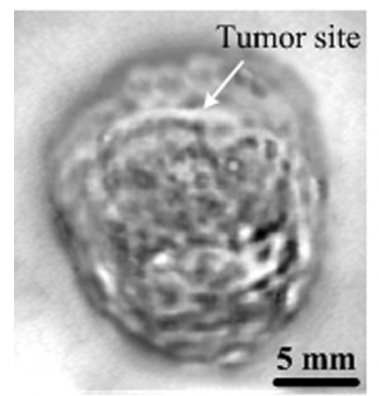

(a)

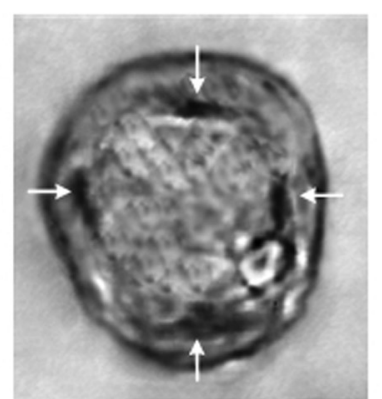

(b)

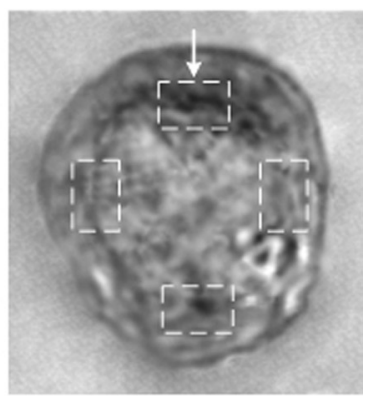

(c)

Fig. 4 Photoacoustic imaging of osteosarcoma (a) before, and (b) $30 \mathrm{~min}$ and (c) $6 \mathrm{~h}$ after the injection of gold nanorods subcutaneously through a needle via four directions. Injected location is indicated by arrows separately in (b), and metabolic lag of gold nanorods in the osteosarcoma region is indicated by an arrow in (c).

tumor site is attributed to the metabolic lag of the gold nanorods in the osteosarcoma region for the EPR effect. ${ }^{15}$ The pixel value of the four areas marked in Fig. 4(c) were analyzed for a statistical comparison between tumorous and normal tissues, and about $87.1 \%$ single enhancement was achieved in the tumor site. The experiment demonstrated that PAI has the potential to identify a tumor by using an extrinsic agent.

\section{Discussion and Conclusion}

In conclusion, this study indicates the feasibility of in vivo osteosarcoma imaging in an animal model by utilizing the hybrid PAI technology. Animal models provide us with convenient tools for research on clinical medical problems. Since PA images were reconstructed of the normal leg and tumorous leg in vivo, the tissue structures, the marked protruding border of the tumor, and the growth trends of the implanted tumor were accurately mapped with time-dependent PAI slices. This imaging technique can be expanded to help delimit the range of the tumor and monitor different tumor stages and development changes through a series of PA images. There is no doubt that PAI is a new approach in the detection and imaging of osteosarcoma. Gold nanorods were used as contrast agents to enhance the PAI detection of osteosarcoma in this study. Metabolic lag in the osteosarcoma region was observed $6 \mathrm{~h}$ after the local injection of gold nanorods. In the future, these particles can be specifically tailored to preferentially accumulate in the tumor after being injected into a patient's bloodstream. This can be achieved by attaching tumor-specific antibodies that will bind only to the relevant cancer cells.

In summary, the noninvasive PAI technology was used in osteosarcoma diagnosis for the first time by imaging osteosarcoma at the early stage and monitoring its development. The in vivo imaging results using a rat model suggest that PAI can be used for mapping osteosarcoma and monitoring its progression. Therefore, PAI technology has a potential application in the diagnosis of osteosarcoma, especially in the early stage osteosarcoma detection, which would in turn lead to beneficial therapeutic effects for osteosarcoma patients.

\section{Acknowledgments}

This research was supported by the National Basic Research Program of China (2010CB732602; 2011CB910402), the Program for Changjiang Scholars and Innovative Research Team in
University (IRT0829), and the National Natural Science Foundation of China (30870676).

\section{References}

1. H. D. Dorfman and B. Czerniak, "Bone cancers," Cancer 75, 203-210 (1995).

2. Piero Picci, "Osteosarcoma: What did we learn from the paediatric experience for adolescents and young adults?," Eur. J. Cancer 5(5), 227-234 (2007).

3. A. A. Kurth and R. Müller, "The effect of an osteolytic tumor on the three-dimensional trabecular bone morphology in an animal model," Skeletal Radiol. 30, 94-98 (2001).

4. G. R. Dickson, C. Geddis, N. Fazzalari, D. Marsh, and I. Parkinson, "Microcomputed tomography imaging in a rat model of delayed union/non-union fracture," J. Orthop. Res. 26, 729-736 (2006).

5. F. Chen, X. Sun, F. de Keyzer, J. Yu, R. Peeters, W. Coudyzer, V. Vandecaveye, W. Landuyt, H. Bosmans, P. van Hecke, G. Marchal, and $\mathrm{Y}$. Ni, "Liver tumor model with implanted rhabdomyosarcoma in rats: MR imaging, microangiography, and histopathologic analysis," Radiology 239, 554-562 (2006).

6. D. Razansky, M. Distel, C. Vinegoni, R. Ma, N. Perrimon, R. W. Köster, and V. Ntziachristos, "Multispectral opto-acoustic tomography of deepseated fluorescent proteins in vivo," Nature Photon. 3, 412-417 (2009).

7. H. F. Zhang, K. Maslov, G. Stoica, and L. H. Wang, "Functional photoacoustic microscopy for high-resolution and noninvasive in vivo imaging" Nature Biotechnol. 24, 848-851 (2006).

8. D. W. Yang, D. Xing, S. H. Yang, and L. Z. Xiang, "Fast full-view photoacoustic imaging by combined scanning with a linear transducer array," Opt. Express 15, 15566-15575 (2007).

9. S. H. Yang, D. Xing, Q. Zhou, L. Z. Xiang, and Y. Q. Lao, "Functional Imaging of Cerebrovascular Activities using High Resolution Photoacoustic Tomography," Med. Phys. 34(8), 3294-3301 (2007).

10. D. L. Chamberland, X. Wang, and B. J. Roessler, "Photoacoustic tomography of carrageenan-induced arthritis in a rat model," J. Biomed. Opt. 13, 011005 (2008).

11. D. L. Chamberland, A. Agarwal, N. Kotov, J. B. Fowlkes, P. L. Carson, and X. D. Wang, "Photoacoustic tomography of joints aided by an Etanercept-conjugated gold nanoparticle contrast agent-an ex vivo preliminary rat study," Nanotechnology 19, 095101 (2008).

12. Y. Q. Lao, D. Xing, S. H. Yang, and L. Z. Xiang, "Noninvasive photoacoustic imaging of the developing vasculature during early tumor growth," Phys. Med. Biol. 53, 4203-4212 (2008).

13. Q. Zhang, N. Iwakuma, P. Sharma, B. M. Moudgil, C. Wu, J. McNeill, H. Jiang, and S. R. Grobmyer, "Gold nanoparticles as a contrast agent for in vivo tumor imaging with photoacoustic tomography," Nanotechnology 20395102 (2009).

14. Z. Yu, H. H. Sun, Q. Y. Fan, H. Long, and B. Ma, "Establishment of reproducible osteosarcoma rat model using orthotopic implantation technique," Onc. Rep. 21, 1175-1180 (2009).

15. H. Maeda, J. Wu, T. Sawa, Y. Matsumura, and K. Hori, "Tumor vascular permeability and the EPR effect in macromolecular therapeutics: a review," J. Cont. Rel. 65, 271-84 (2000). 